\title{
Symmetry-breaking study with deformed ensembles
}

\author{
J. X. de Carvalho, ${ }^{1,2}$ M. S. Hussein,,${ }^{1,2}$ M. P. Pato, ${ }^{2}$ and A. J. Sargeant ${ }^{2}$ \\ ${ }^{1}$ Max-Planck-Institut für Physik komplexer Systeme Nöthnitzer Straße 38, D-01187 Dresden, Germany \\ ${ }^{2}$ Instituto de Física, Universidade de São Paulo, C.P. 66318, 05315-970 São Paulo, S.P., Brazil \\ (Received 28 March 2007; revised manuscript received 14 October 2007; published 27 December 2007)
}

\begin{abstract}
A random matrix model to describe the coupling of $m$-fold symmetry is constructed. The particular threefold case is used to analyze data on eigenfrequencies of elastomechanical vibration of an anisotropic quartz block. It is suggested that such an experimental and theoretical study may supply a powerful means to discern the intrinsic symmetry of physical systems.
\end{abstract}

DOI: 10.1103/PhysRevE.76.066212

PACS number(s): 05.45.Gg, 03.65.Sq, 62.30.+d

The standard ensembles of random matrix theory (RMT) [1] have had wide application in the description of the statistical properties of eigenvalues and eigenfunctions of complex many-body systems. Other ensembles have also been introduced [2] in order to cover situations that depart from universality classes of RMT. One such class of ensembles is the so-called deformed Gaussian orthogonal ensemble (DGOE) [3-6], which proved to be particularly useful when one wants to study the breaking of a discrete symmetry in a many-body system such as the atomic nucleus.

In fact, the use of spectral statistics as a probe of symmetries in physical systems has been a subject of intensive experimental and theoretical investigation following the pioneering work of Bohigas, Giannoni, and Schmit [7] which showed that the quantal behavior of classically chaotic systems exhibits the predictions supplied by the RMT. Examples of symmetry breaking in physical systems that have been studied include nuclei $[8,9]$, atoms $[10,11]$, and mesoscopic devices such as quantum dots [12].

In the case of nuclei, the Mitchell group at the Triangle Universities Nuclear Laboratory [8,9] studied the effect of isospin symmetry breaking in odd-odd nuclei such as ${ }^{26} \mathrm{Al}$. They detected the breakdown of this important symmetry by the applications of two statistics: the short-range, nearestneighbor level spacing distribution (NND) and the longrange Dyson's $\Delta$ statistics $[8,9]$. These results were well described by a DGOE in which a pair of diagonal blocks is coupled. The strength of the coupling needed to account for the symmetry breaking can be traced to the average matrix element of the Coulomb interaction responsible for this discrete symmetry breaking $[4,13]$. The justification for the use of block matrices to describe the statistics of a superposition of $R$ spectra with different values of the conserved quantum number can be traced to Refs. $[1,14]$. In the case of noninteracting spectra-i.e., if the quantum number is exactly conserved-the answer is a superposition of the $R$ spectra. Since the level repulsion is present in each one of the $R$ spectra, their superposition does not show this feature. Thus, we can say that for each spectra of states of a given value of the quantum number, one attaches a random matrix (GOE). For $R$ spectra each of which has a given value of the conserved quantum number, one would have an $R \times R$ block diagonal matrix. Each block matrix will have a dimension dictated by the number state of that spectra. If the quantum number is not conserved, then the $R \times R$ block matrix ac- quires nondiagonal matrices that measure the degree of the breaking of the associated symmetry. This idea was employed by Guhr and Weidenmüller [13] and Hussein and Pato [3] to discuss isopin violation in the nucleus ${ }^{26} \mathrm{Al}$. In Ref. [3], the random block matrix model was called the deformed Gaussian orthogonal ensemble.

In order to study transitions among universal classes of ensembles such as order-chaos (Poisson $\rightarrow$ GOE), symmetry violation transitions $(2 \mathrm{GOE} \rightarrow 1 \mathrm{GOE})$, experiments on physical systems are more complicated due to the difficulty of tuning the interaction (except, e.g., in highly excited atoms where the application of a magnetic field allows the study of GOE-Gaussian unitary ensemble (GUE) transitions). To simulate the microscopic physical systems, one relies on analog computers such as microwave cavities, pioneered by Graf et al. [15] and acoustic resonators of Ellegaard and collaborators [16-18]. It is worth mentioning at this point that the first to draw attention to the applicability of RMT to accoustic waves in physical system was Weaver [19].

In the experiment of Ellegaard et al. [17] what was measured were eigenfrequencies of the elastomechanical vibrations of an anisotropic crystal block with a D3 point-group symmetry. The rectangular crystal block employed by Ellegard et al. was so prepared as to have only a twofold flip symmetry retained. Then, to all effects, the quartz specimen resembles a system of two three-dimensional Sinai billiards. The statistical treatment of the eigenfrequencies of such a block would follow that of the superposition of two uncoupled GOE's.

Then, by removing octants of progressively larger radius from a corner of the crystal block this remnant twofold symmetry was gradually broken. The spectral statistics show a transition towards fully a chaotic system as the octant radius increases. What was then seen was that the measured NND is compatible with a two-block DGOE description but the $\Delta$ statistics was discrepant. This discrepancy was attributed to pseudointegrable behavior, and this explanation was later implemented with the result that the long-range behavior was fitted at the cost, however, of losing the previous agreement shown by the NND [23].

Here we reanalyze this experiment following the simpler idea of extending the DGOE matrix model [5] to consider the coupling of three instead of two GOE's [6]. We show that within this extension both, the short- and long-range statis- 
tics are reasonably fitted, suggesting that the assumption of the reduction of the complex symmetries of anisotropic quartz block may not be correct. Our findings have the potential of supplying very precise means of testing details of symmetry breaking in physical systems.

To define the ensembles of random matrices we are going to work with, we recall the construction based on the maximum entropy principle [3], which leads to a random Hamiltonian which can be cast into the form

$$
H=H_{0}+\lambda H_{1},
$$

where the block diagonal $H_{0}$ is a matrix made of $m$ uncoupled GOE blocks and $\lambda(0 \leqslant \lambda \leqslant 1)$ is the parameter that controls the coupling among the blocks represented by the $H_{1}$ off-diagonal blocks. For $\lambda=1$, the $H_{1}$ part completes the $H_{0}$ part and $H=H^{G O E}$.

These two matrices $H_{0}$ and $H_{1}$ are better expressed introducing the following $m$ projection operators:

$$
P_{i}=\sum_{j \in I_{i}}|j\rangle\langle j|,
$$

where $I_{i}$ defines the domain of variation of the row and column indexes associated with $i$ th diagonal block of size $M_{i}$. Since we are specifically interested in the transition from a set of $m$ uncoupled GOE's to a single GOE, we use the above projectors to generalize our previous model $[3,4]$ by writing

$$
H_{0}=\sum_{i=1}^{m} P_{i} H^{G O E} P_{i}
$$

and

$$
H_{1}=\sum_{i=1}^{m} P_{i} H^{G O E} Q_{i},
$$

where $Q_{i}=1-P_{i}$. It is easily verified that $H=H^{G O E}$ for $\lambda=1$.

The joint probability distribution of matrix elements can be put in the form $[3,20]$

$$
P(H, \alpha, \beta)=Z_{N}^{1} \exp \left(-\alpha t r H^{2}-\beta t r H_{1}^{2}\right),
$$

with the parameter $\lambda$ being given in terms of $\alpha$ and $\beta$ by

$$
\lambda=\left(1+\frac{\beta}{\alpha}\right)^{-1 / 2} .
$$

Statistical measures of the completely uncoupled $m$ blocks have been derived. They show that level repulsion disappears which can be understood since eigenvalues from different blocks behave independently. In fact, as $m$ increases the Poisson statistics are gradually approached. In the interpolating situation of partial coupling, some approximate analytical results have been derived. In Ref. [20], for instance, it has been found that the density $\rho(E)$ for arbitrary $\lambda$ and $m$ is given by

$$
\rho(E)=\sum_{i=1}^{m} \frac{M_{i}}{N} \rho_{i}(E)
$$

where

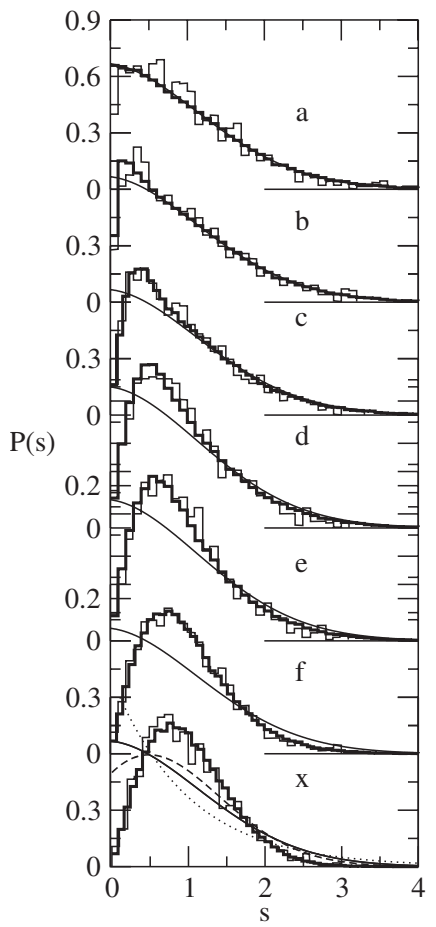

FIG. 1. Nearest-neighbor distributions. Histograms show data (a)-(x) from Ref. [17]. Thick histograms show the three coupled GOE fits to the data carried out using the DGOE numerical simulations using Eq. (5). Also shown as the solid thin line the three uncoupled GOE $P(s)$. In graph $(\mathrm{x})$ the dotted line is the Poisson distribution and the dashed line is the two uncoupled GOE $P(s)$. The very thin line is the Wigner distribution which is hidden behind histograms. The values of $\lambda$ that adjust the data are 0.0032, 0.0071, $0.0158,0.0250,0.0333,0.9950,1.000$ for cases (a)-(x). See text for details.

$$
\rho_{i}(E)= \begin{cases}\frac{2}{\pi a_{i}^{2}} \sqrt{a_{i}^{2}-E^{2}}, & |E| \leqslant a, \\ 0, & |E|>a,\end{cases}
$$

is Wigner's semicircle law with $a=\sqrt{N / \alpha}$ and

$$
a_{i}^{2}=a^{2}\left[\frac{M_{i}}{N}+\lambda^{2}\left(1-\frac{M_{i}}{N}\right)\right] .
$$

Equation (5) can be used to calculate exactly analytically the NND for $2 \times 2$ and $3 \times 3$ matrices [6]. For the $2 \times 2$ case the DGOE, Eq. (5), gives

$$
P_{2 \times 2}(s, \beta)=\alpha s \exp \left(-\frac{\alpha}{2} s^{2}\right) \sqrt{1+\frac{\beta}{\alpha}} I_{0}\left(\frac{\beta s^{2}}{4}\right) \exp \left(-\frac{\beta s^{2}}{4}\right),
$$

where $I_{0}$ is the modified Bessel function, whose asymptotic form is

$$
I_{0}(x) \rightarrow \frac{e^{x}}{\sqrt{2 \pi x}} .
$$

Thus; there is no level repulsion for $\beta \rightarrow \infty, P_{2 \times 2}(s, \infty)$ $=\frac{2}{\pi} \alpha \exp \left(-\frac{\alpha}{2} s^{2}\right)$, which represents the $2 \times 2$ Poisson distribu- 


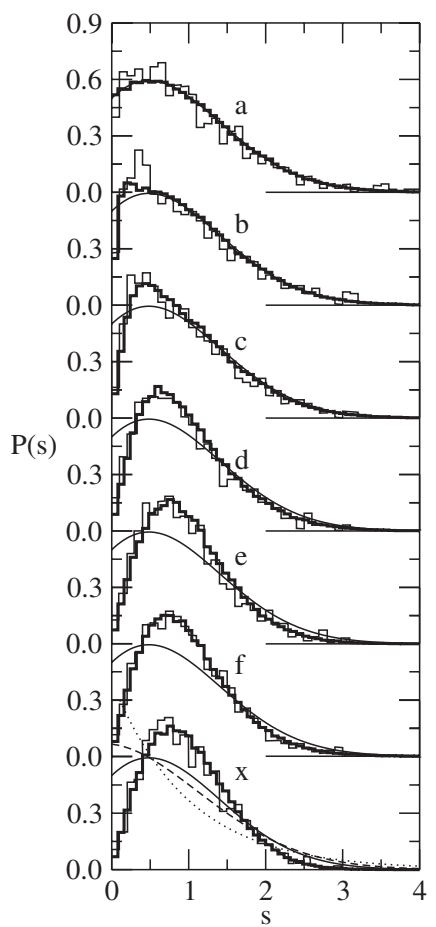

FIG. 2. Nearest-neighbor distributions. Histograms show data (a)-(x) from Ref. [17]. Thick histograms show the two coupled GOE fits to the data carried out using the DGOE numerical simulations using Eq. (5). Also shown as the solid thin line are the two uncoupled GOE $P(s)$. In graph $(\mathrm{x})$ the dotted line is the Poisson distribution and the dashed line is three uncoupled GOE $P(s)$. The very thin line is the Wigner distribution which is hidden behind histograms. The values of $\lambda$ that adjust the data are $0.000,0.0258$, $0.0200,0.0400,0.0705,0.0600,1.000$ for cases (a)-(x). See text for details.

tion where the usual exponential is replaced by a Gaussian. The prefactor is just 1 if $2 \alpha$ is taken to be $\pi$. In the opposite limit, $\beta \rightarrow 0, I_{0}(x) \approx 1-x^{2} / 4$ and one obtains the Wigner distribution

$$
P_{2 \times 2}(s, \beta \rightarrow 0) \approx \frac{\pi}{2} \exp \left(-\frac{\pi}{4} s^{2}\right) s
$$

Note that the parameter $\lambda$ of Eq. (6) is 0 if $\beta$ is $\infty$ and 1 if $\beta$ is 0 .

For higher dimensions Eq. (5) can only be used for numerical simulations. This is what we are now reporting, using two- and three-block matrices of sizes $105 \times 105$ and $70 \times 70$ each, respectively. The size of the whole matrix is $210 \times 210$. Further, we take an ensemble of 1000 elements and fix $\alpha$ to be 1 . We apply our model to analyze the eigenfrequency data of the elastomechanical vibrations of an anisotropic quartz block used in [17].

In this reference in order to break the flip symmetry of the crystal block gradually they removed an octant of a sphere of varying size at one of the corners. The rectangular quartz block has the dimensions $14 \times 25 \times 40 \mathrm{~mm}^{3}$. The radii of the spheres containing the octants are $r=0.0,0.5,0.8,1.1,1.4$, and $1.7 \mathrm{~mm}$, representing figures (a)-(f). Figures $1 x$ and $2 x$

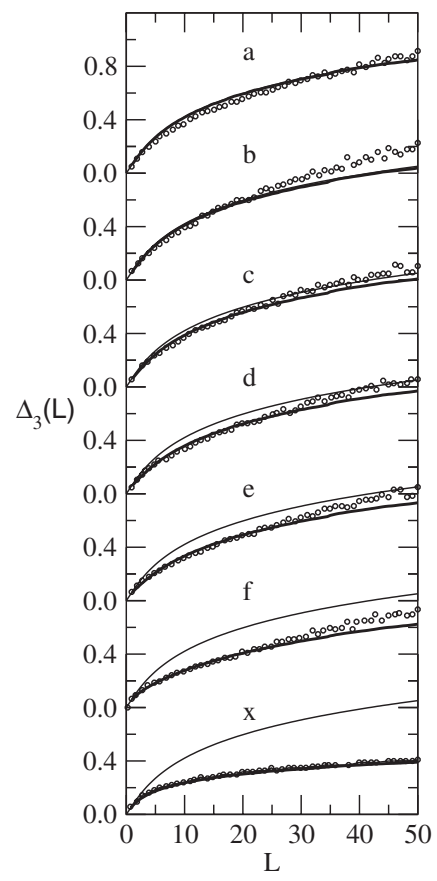

FIG. 3. Spectral rigidities. The thick lines are the DGOE simulation for the three coupled GOE's. The same values of $\lambda$ as in Fig. 1 were used. The thin lines correspond to the three uncoupled GOE's case. The data points are from Ref. [17]. See text for details.

of Ref. [17] correspond to an octant of a huge sphere of radius $r=10.0 \mathrm{~mm}$, whose center is inside the crystal and close to one of the corners. They found 1424, 1414, 1424, 1414,1424 , and 1419 frequency eigenmodes in cases (a)-(f), respectively. These eigenfrequecies were measured in the frequency range between 600 and $900 \mathrm{kHz}$. Thus the average spacing between the modes is about $214 \mathrm{~Hz}$. The histograms and circles in the two figures of Ref. [17] represent the shortrange nearest-neighbor distributions $P(s)$ (Fig. 1) and the long-range $\Delta_{3}(L)$ statistics (Fig. 2). In our DGOE simulation the unfolding of the calculated spectra is performed with the DGOE density given by Eq. (7) above.

In Figs. 1 and 2, we show the results of our simulations as compared to the data of Ellegaard et al. [17] for the spacing distribution and in Figs. 3 and 4 the long-range correlation exemplified by the spectral rigidity $\Delta_{3}(L)$. We simulate the gradual breaking of the twofold or threefold symmetry by changing the value of the parameter $\lambda$ above. We see clearly that in so far as the $\Delta_{3}(L)$ is concerned a $3 \mathrm{GOE}$ description works much better than a $2 \mathrm{GOE}$ one. It is clear, however that both descriptions fall below the data, especially at large $L$. We shall analyze this discrepancy in the following using the missing level effect [21].

It is often the case that there are some missing levels in the statistical sample analyzed. Such a situation was addressed recently by Bohigas and Pato [21] who have shown that if $g$ fraction of the levels or eigenfrequencies is missing, the $\Delta_{3}(L)$ becomes

$$
\Delta_{3}^{g}(L)=g \frac{L}{15}+(1-g)^{2} \Delta_{3}\left(\frac{L}{1-g}\right) .
$$

The presence of the linear term, even if small, could explain 


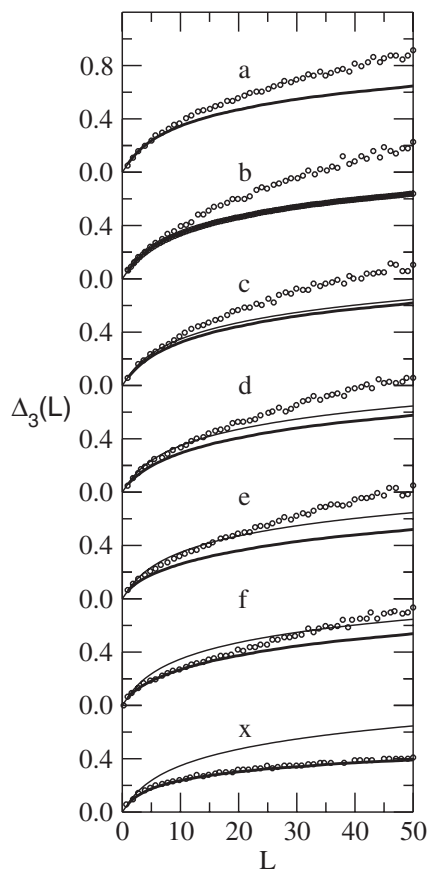

FIG. 4. Spectral rigidities. The thick lines are the DGOE simulation for the two coupled GOE's. The same values of $\lambda$ as in Fig. 2 were used. The thin lines correspond to the two uncoupled GOE's case. The data points are from Ref. [17]. See text for details.

the large- $L$ behavior of the measured $\Delta_{3}(L)$. We call this effect the missing level (ML) effect. Another possible deviation of $\Delta_{3}$ could arise from the presence of pseudointegrable (PI) effect $[22,23]$. This also modifies $\Delta_{3}$ by adding a Poisson term just like Eq. (13). In the following we show that there is no need for the PI effects to explain the large- $L$ data on the $\Delta_{3}$ if the ML effect is taken into account.

We take a study case, Figs. 3(b) and 4(b), which correspond to $r=0.5 \mathrm{~mm}$ and where 1414 frequency eigenvalues were found. We consider this a potential ML case and take for $\Delta_{3}$ the expression given in Eq. (13) and apply to our simulations. We find a perfect fit to the data if $g$ is taken to be 0.1 ; namely, only $90 \%$ of the eigenfrequencies were in fact taken into account in the statistical analysis. There is, therefore, room to account much better for all cases [Figs. 2(a), 2(c), etc.] by appropriately choosing the corresponding value of $g$. We have also verified that if a $2 \mathrm{GOE}$ description is used-namely, $m=2$ - then an account of the large- $L$ behavior of $\Delta_{3}$ can also be obtained if a much larger number of levels were missing in the sample. In our particular case of Fig. 2(b), we obtained $g=0.3$. This is 3 times larger than the ML needed in the $3 \mathrm{GOE}$ description. We consider the large value of $g$ needed in the $2 \mathrm{GOE}$ description much too large to

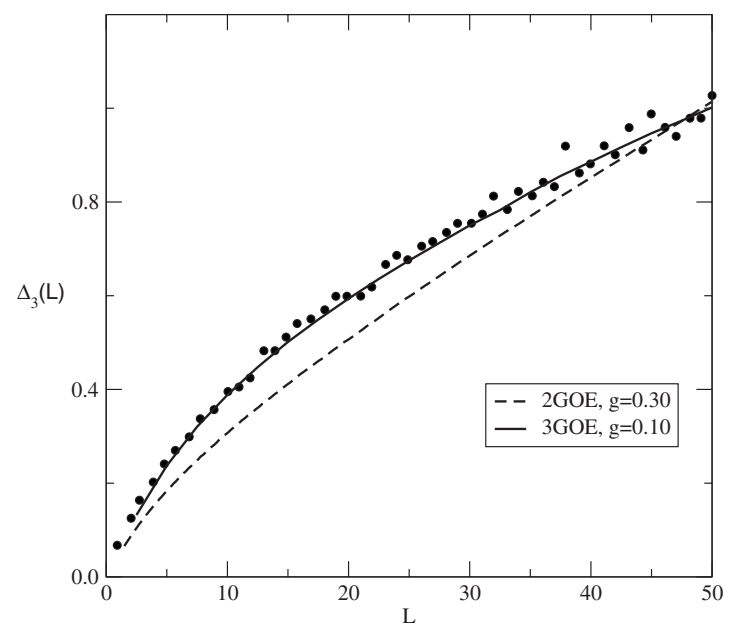

FIG. 5. The ML effect. The data points correspond to case (b) of Ref. [17], $r=0.5 \mathrm{~mm}$. The solid line corresponds to our three coupled GOE's fit with $\lambda=0.0071$, Fig. 3(b), and $g=0.10$. The dashed line corresponds to our two coupled GOE's fit with $\lambda$ $=0.0258$, Fig. $4(\mathrm{~b})$, and $g=0.30$. See text for details.

conform to the reported data in Ref [17]. Figure 5 summarizes the above.

It is therefore clear that the $3 \mathrm{GOE}$ description of the spectral rigidity of the eigenfrequency spectra of [17] for the crystal block does work very well if a small fraction of the levels is taken to be missing, without resort to pseudointegrable trajectories or levels that do not feel the symmetry breaking [23]. On the other hand, the 2GOE description, which does as good as the $3 \mathrm{GOE}$ one in fitting the measured $P(s)$, fails dramatically in accounting for the spectral rigidity, even if as much as $30 \%$ of the levels are taken as missing.

In conclusion, a random matrix model to describe the coupling of $m$-fold symmetry is constructed. The particular threefold case is used to analyze data on eigenfrequencies of elastomechanical vibration of a anisotropic quartz block. By properly taking into account the ML effect we have shown that the quartz block could very well be described by three uncoupled GOE's, which are gradually coupled by the break of the threefold symmetry (through the gradual removal of octants of increasing sizes), until a $1 \mathrm{GOE}$ situation is attained. This, therefore, indicates that the unperturbed quartz block may possess another symmetry besides the flip one. A preliminary version of the formal aspect of this work has previously appeared in [24].

This work was supported in part by the $\mathrm{CNPq}$ and FAPESP (Brazil). M. S. H. was supported by the Martin Gutzwiller Program at the MPIPKS-Dresden 2007/2008.
[1] M. L. Mehta, Random Matrices, 2nd ed. (Academic Press, Boston, 1991); T. A. Brody et al., Rev. Mod. Phys. 53, 385 (1981); T. Guhr, A. Müller-Groeling, and A. Weidenmüller, Phys. Rep. 299, 189 (1998).
[2] F. J. Dyson, J. Math. Phys. 3, 1191 (1962).

[3] M. S. Hussein and M. P. Pato, Phys. Rev. Lett. 70, 1089 (1993).

[4] M. S. Hussein and M. P. Pato, Phys. Rev. C 47, 2401 (1993). 
[5] M. S. Hussein and M. P. Pato, Phys. Rev. Lett. 80, 1003 (1998).

[6] C. E. Carneiro, M. S. Hussein, and M. P. Pato, in Quantum Chaos, edited by H. A. Cerdeira, R. Ramaswamy, M. C. Gutzwiller, and G. Casati (World Scientific, Singapore, 1991), p. 190.

[7] O. Bohigas, M. J. Giannoni, and C. Schmit, Phys. Rev. Lett. 52, 1 (1984). See also O. Bohigas and M. J. Giannoni, in Mathematical and Computational Methods in Nuclear Physics, edited by J. S. DeHesa, J. M. Gomez, and A. Polls, Lecture Notes in Physics, Vol. 209 (Springer-Verlag, New York, 1984).

[8] G. E. Mitchell, E. G. Bilpuch, P. M. Endt, and J. F. Shriner, Phys. Rev. Lett. 61, 1473 (1988).

[9] A. A. Adams, G. E. Mitchell, and J. F. Shriner, Jr., Phys. Lett. B 422, 13 (1998).

[10] B. D. Simons, A. Hashimoto, M. Courtney, D. Kleppner, and B. L. Altshuler, Phys. Rev. Lett. 71, 2899 (1993).

[11] G. R. Welch, M. M. Kash, C-h Iu, L. Hsu, and D. Kleppner, Phys. Rev. Lett. 62, 893 (1989).

[12] See, e.g., Y. Alhassid, Rev. Mod. Phys. 72, 895 (2000) and references therein.

[13] T. Guhr and H. A. Weidenmüller, Ann. Phys. (N.Y.) 199, 412 (1990).

[14] N. Resenzweig and C. E. Porter, Phys. Rev. 120, 1698 (1960).

[15] H.-D. Graf, H. L. Harney, H. Lengeler, C. H. Lewenkopf, C. Rangacharyulu, A. Richter, P. Schardt, and H. A. Weidenmuller, Phys. Rev. Lett. 69, 1296 (1992); H. Alt, H.-D. Graf, H. L. Harney, R. Hofferbert, H. Lengeler, A. Richter, P. Schardt, and H. A. Weidenmuller, ibid. 74, 62 (1995); H. Alt, C. Dembowski, H.-D. Graf, R. Hofferbert, H. Rehfeld, A. Richter, R. Schuhmann, and T. Weiland, ibid. 79, 1026 (1997); H. Alt, C. I. Brabosa, H.-D. Graf, T. Guhr, H. L. Harney, R. Hofferbert, H. Rehfeld, and A. Richter, ibid. 81, 4847 (1998); C. Dembowski, H.-D. Graf, A. Heine, R. Hofferbert, H. Reh- feld, and A. Richter, ibid. 84, 867 (2000); C. Dembowski, H.-D. Graf, H. L. Harney, A. Heine, W. D. Heiss, H. Rehfeld, and A. Richter, ibid. 86, 787 (2001); C. Dembowski, H.-D. Graf, A. Heine, T. Hesse, H. Rehfeld, and A. Richter, ibid. 86, 3284 (2001); C. Dembowski, B. Dietz, H.-D. Graf, A. Heine, T. Papenbrock, A. Richter, and C. Richter, ibid. 89, 064101 (2002); C. Dembowski, B. Dietz, H.-D. Graf, A. Heine, F. Leyvraz, M. Miski-Oglu, A. Richter, and T. H. Seligman, ibid. 90, 014102 (2003); C. Dembowski, B. Dietz, H.-D. Graf, H. L. Harney, A. Heine, W. D. Heiss, and A. Richter, ibid. 90, 034101 (2003); C. Dembowski, B. Dietz, T. Friedrich, H.-D. Graf, A. Heine, C. Mejia-Monasterio, M. Miski-Oglu, A. Richter, and T. H. Seligman, ibid. 93, 134102 (2004); B. Dietz, T. Guhr, H. L. Harney, and A. Richter, ibid. 96, 254101 (2006); E. Bogomolny, B. Dietz, T. Friedrich, M. Miski-Oglu, A. Richter, F. Schafer, and C. Schmit, ibid. 97, 254102 (2006); B. Dietz, T. Friedrich, H. L. Harney, M. Miski-Oglu, A. Richter, F. Schafer, and H. A. Weidenmuller, ibid. 98, 074103 (2007).

[16] C. Ellegaard, T. Guhr, K. Lindemann, H. Q. Lorensen, J. Nygard, and M. Oxborrow, Phys. Rev. Lett. 75, 1546 (1995).

[17] C. Ellegaard, T. Guhr, K. Lindemann, J. Nygard, and M. Oxborrow, Phys. Rev. Lett. 77, 4918 (1996).

[18] P. Bertelsen, C. Ellegaard, T. Guhr, M. Oxborrow, and K. Schaadt, Phys. Rev. Lett. 83, 2171 (1999).

[19] R. L. Weaver, J. Acoust. Soc. Am. 85, 1005 (1989).

[20] A. C. Bertuola, J. X. de Carvalho, M. S. Hussein, M. P. Pato, and A. J. Sargeant, Phys. Rev. E 71, 036117 (2005).

[21] O. Bohigas and M. P. Pato, Phys. Lett. B 595, 171 (2004).

[22] D. Biswas and S. R. Jain, Phys. Rev. A 42, 3170 (1990).

[23] A. Abd El-Hady, A. Y. Abul-Magd, and M. H. Simbel, J. Phys. A 35, 2361 (2002).

[24] M. S. Hussein, J. X. de Carvalho, M. P. Pato, and A. J. Sargeant, Few-Body Syst. 38, 209 (2006). 